\title{
Hyperthermic intraoperative pleural cisplatin chemotherapy extends interval to recurrence and survival among low-risk patients with malignant pleural mesothelioma undergoing surgical macroscopic complete resection
}

\author{
David J. Sugarbaker, MD, ${ }^{\mathrm{a}}$ Ritu R. Gill, MD, ${ }^{\mathrm{b}}$ Beow Y. Yeap, $\mathrm{ScD},{ }^{\mathrm{c}}$ Andrea S. Wolf, MD, MPH, ${ }^{\mathrm{a}}$ \\ Marcelo C. DaSilva, MD, ${ }^{\mathrm{a}}$ Elizabeth H. Baldini, MD, MPH, ${ }^{\mathrm{d}}$ Raphael Bueno, MD, ${ }^{\mathrm{a}}$ and \\ William G. Richards, $\mathrm{PhD}^{\mathrm{a}}$
}

\begin{abstract}
Objective: Local recurrence limits long-term survival in patients with malignant pleural mesothelioma. We investigated whether hyperthermic intraoperative cisplatin chemotherapy lavage affects the interval to recurrence and overall survival among patients with favorable prognostic factors.
\end{abstract}

\begin{abstract}
Methods: Using a preoperative risk assessment algorithm we had previously developed and validated, we retrospectively identified a cohort of patients treated with cytoreductive surgery from 2001 to 2009 . The patients had epithelial histologic findings on biopsy and were characterized as having a low risk of early recurrence and death (ie, tumor volume $\leq 500 \mathrm{~cm}^{3}$ and were either men with a hemoglobin level of $\geq 13 \mathrm{~g} / \mathrm{dL}$ or were women). Those patients who had received hyperthermic intraoperative cisplatin chemotherapy were compared with a comparison group of those who had not. Fisher's exact test was used to determine the balance of prognostic factors. The Kaplan-Meier method and log-rank tests were used to estimate and compare the interval to recurrence and overall survival. Cox proportional hazards regression was used for multivariate analysis.
\end{abstract}

\begin{abstract}
Results: The cohort criteria identified 103 patients: 72 who received hyperthermic intraoperative cisplatin chemotherapy and 31 who did not. The groups were balanced for prognostic factors, except for the use of neoadjuvant chemotherapy (more common in the comparison group). The hyperthermic intraoperative cisplatin chemotherapy group exhibited a significantly longer interval to recurrence (27.1 vs 12.8 months) and overall survival (35.3 vs 22.8 months) than the comparison group. The improved interval to recurrence and overall survival for the hyperthermic intraoperative cisplatin chemotherapy group were particularly evident among the subgroups of patients who had not received hemithoracic radiotherapy and who had pathologic stage N1 or N2 lymph node metastases.
\end{abstract}

Conclusions: A favorable outcome and minimal incremental morbidity support the incorporation of hyperthermic intraoperative cisplatin chemotherapy into multimodality treatment strategies for patients with low-risk epithelial malignant pleural mesothelioma. (J Thorac Cardiovasc Surg 2013;145:955-63)

\begin{abstract}
Malignant pleural mesothelioma (MPM) is a rare, locally aggressive, and rapidly fatal tumor of the thoracic pleural mesothelium commonly associated with inhalational exposure to asbestos. It is refractory to single modality local or systemic therapy. Multimodality approaches involving

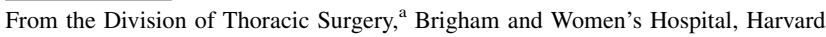
Medical School, Boston, Mass; Department of Radiology, ${ }^{\mathrm{b}}$ Brigham and Women's Hospital, Harvard Medical School, Boston, Mass; Department of Medicine, ${ }^{\mathrm{c}}$ Massachusetts General Hospital, Harvard Medical School, Boston, Mass; and Department of Radiation Oncology, ${ }^{\mathrm{d}}$ Brigham and Women's Hospital, Dana-Farber Cancer Institute, Harvard Medical School, Boston, Mass.

Disclosures: Authors have nothing to disclose with regard to commercial support.

Received for publication April 30, 2012; revisions received Nov 26, 2012; accepted for publication Dec 11, 2012.

Address for reprints: David J. Sugarbaker, MD, Division of Thoracic Surgery, Brigham and Women's Hospital, 75 Francis St, Boston, MA 02115 (E-mail: dsugarbaker@partners.org).

0022-5223/\$36.00

Copyright (C) 2013 Published by Elsevier Inc. on behalf of The American Association for Thoracic Surgery

http://dx.doi.org/10.1016/j.jtcvs.2012.12.037
}

surgical macroscopic complete resection ${ }^{1}$ using either extrapleural pneumonectomy combined with chemotherapy and radiotherapy or pleurectomy/decortication combined with chemotherapy have been associated with longduration disease remission and overall survival for selected patients. $^{2}$ The tendency for eventual relapse to occur locally in most cases ${ }^{3}$ has prompted us and others to investigate the use of additional modalities to improve local control. During the past decade, we have completed a series of phase I and II trials of hyperthermic intraoperative chemotherapy (HIOC) lavage, initially using cisplatin as a single agent. ${ }^{4-6}$ In the present retrospective study, we sought to determine whether this intervention had a measurable effect on patient outcomes.

The outcomes for patients with MPM who undergo surgical resection have varied, reflecting the heterogeneity of this tumor with regard to the prognostic factors, including gender, ${ }^{7,8}$ histologic features, ${ }^{9}$ tumor burden, anemia, and the 


\section{Abbreviations and Acronyms}

$\mathrm{CT}=$ computed tomography

HIOC $=$ hyperthermic intraoperative cisplatin chemotherapy

MPM $=$ malignant pleural mesothelioma

delivery of adjunctive treatments. ${ }^{10}$ To minimize competing sources of variance and thereby improve the likelihood of detecting an effect of a single dose of intraoperative cisplatin monotherapy, we identified a cohort of patients at low risk of early recurrence and death after macroscopic complete resection and multimodality therapy. Previously, we developed and validated a preoperative risk assessment algorithm that includes the computed tomography (CT)-derived tumor volume, hemoglobin level, and gender for patients with epithelial MPM diagnosed using pleural biopsy. ${ }^{11}$ This algorithm was applied retrospectively to identify, for the present analysis, a cohort of patients with a low preoperative risk of early recurrence and death. Within this cohort, we investigated whether patients who had received HIOC demonstrated a longer interval to recurrence and/or overall survival compared with patients who had not.

\section{METHODS}

With institutional review board approval (protocol no. 2005-p-001520), a study cohort was selected to include all patients within the Brigham and Women's Hospital International Mesothelioma Program Patient Data Registry who (1) had epithelial MPM as determined by biopsy; (2) had undergone intended multimodality therapy from 2001 to 2009, involving macroscopic complete resection with neoadjuvant or adjuvant intravenous chemotherapy and/or radiotherapy; (3) had complete data available for risk assessment (preoperative tissue biopsy findings, preoperative CT scan available electronically for tumor volume calculation, preoperative laboratory findings, gender) and outcomes analysis (MPM recurrence and vital status follow-up); and (4) were categorized as preoperative low risk (defined as epithelial histologic features by biopsy, computed tumor volume $\leq 500 \mathrm{~cm}^{3}$, and either male gender with a hemoglobin level of $\geq 13 \mathrm{~g} / \mathrm{dL}$ or female gender). ${ }^{11}$ The cohort was divided into 2 groups according to whether HIOC had been administered during the operative procedure after tumor removal. The patients who received HIOC were treated using 1 of 4 treatment protocols active during the portions of the study period (protocol 99-124 ${ }^{4}$ [1 patient], protocol 01-116 ${ }^{5}$ [5 patients], protocol $03-302^{6}$ [ 24 patients], and protocol 04-063 [2 patients]) or off protocol using protocol-established safe and effective dose and administration parameters. These parameters included macroscopic complete resection followed by cisplatin 175 to $225 \mathrm{mg} / \mathrm{m}^{2}$ for a 1-hour lavage at $42^{\circ} \mathrm{C}$ with sodium thiosulfate rescue and/or amifostine protection., ${ }^{4,6}$ The control patients had undergone macroscopic complete resection and intended adjuvant and/or neoadjuvant therapy but had not received cisplatin HIOC.

Preoperatively, all patients underwent pleural biopsy and CT imaging. Most also underwent cervical mediastinoscopy. Some patients with positive mediastinal nodal disease on preoperative mediastinoscopy were referred for induction chemotherapy. Other patients had received chemotherapy before referral. The choice of operative procedure was determined for each patient from the preoperative assessment findings. Pleurectomy/ decortication was recommended for patients in whom that procedure was judged likely to obtain macroscopic complete resection-generally patients with low-volume tumor not apparently involving the lung parenchyma and/or fissures - and those patients with contraindications to pneumonectomy. The follow-up schedule for all patients consisted of several visits in the early postoperative period, once every 4 months with CT for 3 years, and every 6 months with CT thereafter. The patients who developed symptoms between the surveillance visits were evaluated with additional imaging, as appropriate.

Categorical or dichotomized continuous variables representing demographic, clinical, and pathologic covariates were assessed for the proportionality of the distribution between the HIOC and comparison groups using Fisher's exact test. In addition to the variables defining low risk (histologic subtype by biopsy, CT-derived tumor volume, gender, and hemoglobin level), the histologic subtype found on the final pathologic examination, pathologic lymph node status, stage, age, length of hospital stay, administration of adjuvant and/or neoadjuvant therapy, surgical procedure, and perioperative mortality were also assessed.

A central review of all preoperative radiographic studies and available studies for evaluation of recurrence status (82 patients [80\%]) was performed by a thoracic radiologist (R.G.). The recurrence-free interval was calculated as the interval from surgery to the first radiographic recurrence or biopsy determination of recurrence, with censoring on the date of the most recent study if recurrence was not demonstrated, the patient was subsequently lost to follow-up, or the date of death if other-cause death was documented (eg, no recurrent mesothelioma at autopsy). Overall survival was calculated as the interval from surgery to death from any cause, with censoring for living patients at the most recent contact date. KaplanMeier survival function estimates were calculated and compared using the log-rank statistic. Multivariate analysis was performed using Cox proportional hazards regression.

\section{RESULTS}

From 2001 through 2009, 616 patients underwent macroscopic complete resection by either extrapleural pneumonectomy or pleurectomy/decortication. Of these, 303 patients (207 of whom received cisplatin HIOC) had the preoperative hemoglobin level and CT data available for tumor volume calculation and could thus be evaluated retrospectively for preoperative risk. The demographic, clinical, treatment, and pathologic characteristics of the 103 patients who had epithelial MPM diagnosed by the biopsy findings and were characterized as low risk, thereby fitting the criteria for inclusion in the study cohort, are listed in Table 1. The low-risk patients who underwent extrapleural pneumonectomy and could be pathologically staged using the International Mesothelioma Interest Group TNM criteria ${ }^{12}$ included 1 patient with stage I $(1 \%), 13$ with stage II $(18 \%), 39$ with stage III $(53 \%)$, and 21 with stage IV (28\%). The median interval to recurrence for the low-risk cohort was 23.6 months after surgery, and the median overall survival was 33.1 months.

Among the 103 low-risk patients, 72 received cisplatin HIOC and constituted the treatment group, and 31 underwent macroscopic complete resection without HIOC and constituted the comparison group. The decision to treat with HIOC was determined on an individual basis. The most common reason that comparison patients did not receive HIOC was previous administration of neoadjuvant platinum-containing chemotherapy (13 patients). The groups were not balanced in this respect- $42 \%$ of the 
TABLE 1. Demographic, clinical, treatment, and pathologic characteristics

\begin{tabular}{|c|c|c|c|c|}
\hline Characteristic & Cohort & HIOC group & Comparison group & $P$ value \\
\hline Patients (n) & 103 & 72 & 31 & \\
\hline \multicolumn{5}{|l|}{ Age (y) } \\
\hline Median & 62 & 61 & 66 & \\
\hline Range & $27-84$ & $27-79$ & $45-84$ & \\
\hline Age $\geq 62$ y & - & $33(46 \%)$ & $20(65 \%)$ & .09 \\
\hline Male gender (n) & $68(66 \%)$ & $48(67 \%)$ & $20(65 \%)$ & .82 \\
\hline Surgical procedure (n) & & & & .63 \\
\hline EPP & $74(72 \%)$ & $53(74 \%)$ & $21(68 \%)$ & \\
\hline $\mathrm{P} / \mathrm{D}$ & $29(28 \%)$ & $19(26 \%)$ & $10(32 \%)$ & \\
\hline Right side (n) & $47(46 \%)$ & $30(42 \%)$ & $17(55 \%)$ & .28 \\
\hline Pathologic subtype (n) & & & & .24 \\
\hline Epithelial & $87(84 \%)$ & $63(88 \%)$ & $24(77 \%)$ & \\
\hline Biphasic & $16(16 \%)$ & $9(13 \%)$ & $7(23 \%)$ & \\
\hline Pathologic lymph node status (n) & & & & $>.99$ \\
\hline $\mathrm{Nx}$ & $23(22 \%)$ & $15(21 \%)$ & $8(26 \%)$ & \\
\hline No & $37(36 \%)$ & $26(36 \%)$ & $11(35 \%)$ & \\
\hline N1 & $19(18 \%)$ & $15(21 \%)$ & $4(13 \%)$ & \\
\hline $\mathrm{N} 2$ & $23(22 \%)$ & $15(21 \%)$ & $8(26 \%)$ & \\
\hline $\mathrm{N}+$ (station unspecified) & $1(1 \%)$ & $1(1 \%)$ & 0 & \\
\hline Node positive (N1, N2, N+ vs N0) & - & $31(55 \%)$ & $12(52 \%)$ & \\
\hline Pathologic TNM stage (EPP only) (n) & & & & .52 \\
\hline I & $1(1 \%)$ & $1(1 \%)$ & 0 & \\
\hline II & $13(18 \%)$ & $8(15 \%)$ & $5(24 \%)$ & \\
\hline III & $39(53 \%)$ & $29(55 \%)$ & $10(48 \%)$ & \\
\hline IV & $21(28 \%)$ & $15(28 \%)$ & $6(29 \%)$ & \\
\hline Stage III-IV (vs I-II) & - & $44(83 \%)$ & $16(76 \%)$ & \\
\hline \multicolumn{5}{|l|}{ Length of hospital stay (d) } \\
\hline Median & 12 & 11 & 12 & \\
\hline Range & $6-84$ & 6-84 & 6-62 & \\
\hline Length of stay $\geq 12 \mathrm{~d}$ & & $34(47 \%)$ & $18(58 \%)$ & .39 \\
\hline Neoadjuvant chemotherapy (n) & $23(22 \%)$ & $10(14 \%)$ & $13(42 \%)$ & .0037 \\
\hline Adjuvant chemotherapy (n) & & & & .13 \\
\hline Unknown (lost to follow-up) & $1(1 \%)$ & 0 & $1(3 \%)$ & \\
\hline No adjuvant chemotherapy & $49(48 \%)$ & $31(43 \%)$ & $18(58 \%)$ & \\
\hline Adjuvant chemotherapy & $53(51 \%)$ & $41(57 \%)$ & $12(39 \%)$ & \\
\hline Adjuvant radiotherapy $(\mathrm{n})$ & & & & .28 \\
\hline Unknown (lost to follow-up) & $1(1 \%)$ & 0 & $1(3 \%)$ & \\
\hline No adjuvant radiotherapy & $48(47 \%)$ & $31(43 \%)$ & $17(52 \%)$ & \\
\hline Adjuvant radiotherapy & $54(52 \%)$ & $41(57 \%)$ & $13(45 \%)$ & \\
\hline Perioperative (30-d or in-hospital) mortality (n) & $3(3 \%)$ & $3(4 \%)$ & 0 & .55 \\
\hline \multicolumn{5}{|l|}{ Preoperative hemoglobin level (g/dL) } \\
\hline Median & 13.8 & 13.9 & 13.6 & \\
\hline Range & $8.7-16.6$ & $8.7-16.6$ & $10.0-16.1$ & \\
\hline Preoperative hemoglobin level $\leq 13.8 \mathrm{~g} / \mathrm{dL}$ & & $36(50 \%)$ & $19(61 \%)$ & .39 \\
\hline \multicolumn{5}{|l|}{ Preoperative tumor volume $\left(\mathrm{cm}^{3}\right)$} \\
\hline Median & 112 & 107 & 146 & \\
\hline Range & $1-478$ & $1-478$ & $4-470$ & \\
\hline Preoperative tumor volume $\geq 112 \mathrm{~cm}^{3}$ & & $34(47 \%)$ & $17(55 \%)$ & .67 \\
\hline
\end{tabular}

patients in the comparison group had received induction platinum-based chemotherapy compared with $14 \%$ of patients in the HIOC group $(P=.0037)$. Most commonly, neoadjuvant chemotherapy consisted of pemetrexed plus cisplatin (18 patients) for a median of 4 cycles (range, 1-8), and 2 of these patients also received other chemotherapy regimens before surgery. Four patients received pemetrexed plus carboplatin for a median of 6 cycles (range, 4-6); 
for 1 patient, the details of neoadjuvant chemotherapy were unknown. Other reasons for not receiving HIOC included borderline renal function and patient choice.

All other demographic, clinical, and pathologic variables with established or potential influence on recurrence and/or survival endpoints were distributed proportionally between the 2 groups (Table 1). These included gender, ${ }^{8}$ age, ${ }^{7}$ surgical procedure, administration of adjuvant chemotherapy and/or radiotherapy (Table 2), ${ }^{10}$ pathologic World Health Organization subtype, ${ }^{13}$ lymph node status, ${ }^{14}$ and International Mesothelioma Interest Group/American Joint Committee on Cancer/International Union Against Cancer TNM stage. ${ }^{12}$

The groups also did not differ significantly in terms of the hospital length of stay (as a surrogate measure of postoperative morbidity) or perioperative mortality. Although not statistically significant, the only 3 postoperative deaths occurred in the HIOC cohort. None of these were thought to be related to the HIOC. Specifically, 1 patient had constrictive pericarditis, 1 died of bronchopleural fistula/empyema, and 1 died of an intracranial hemorrhage after a prolonged intensive care unit stay with multiorgan dysfunction after intraoperative right ventricular failure.

At the analysis, 45 of $72(63 \%)$ patients who had received HIOC had experienced MPM recurrence. Of the 72 patients in the HIOC group, $27(38 \%)$ were censored for recurrence. Of these 27 patients, $13(18 \%)$ had died of other causes, including 3 perioperative deaths $(4.2 \%)$, $3(4.2 \%)$ were lost to follow-up and died with an unknown recurrence status, and $11(15 \%)$ remained alive in active follow-up without evidence of recurrence at a median postoperative interval of 54 months (range, 26-94 months). Of the 31 comparison patients, 23 (74\%) developed recurrence, and $8(26 \%)$ were censored: $4(13 \%)$ were lost to follow-up, and 4 (13\%) died of other causes. Three comparison group patients $(10 \%)$ were alive (with recurrence) at a median postoperative interval of 39 months (range, 38-75 months).

TABLE 2. Details of adjuvant chemotherapy and radiotherapy administered to cohort patients

\begin{tabular}{lcc}
\hline \multicolumn{1}{c}{ Variable } & Patients (n) & Median (range) \\
\hline Adjuvant chemotherapy & & Cycles (n) \\
Cisplatin (concurrent with RT) & 3 & $4(3-4)$ \\
Cisplatin + gemcitabine & 2 & $1.5(1-2)$ \\
Cisplatin + pemetrexed & 39 & $4(1-6)$ \\
Carboplatin + pemetrexed & 6 & $4(3-9)$ \\
Unspecified & 3 & - \\
Adjuvant radiotherapy & & Total dose (cGy) \\
AP/PA & 1 & 5400 \\
MSKCC & 18 & $5400(3420-5400)$ \\
IMRT & 9 & $4860(4500-5400)$ \\
Unspecified & 26 & - \\
\hline
\end{tabular}

$R T$, Radiotherapy; AP/PA, anteroposterior/posteroanterior (fields); $M S K C C$, Memorial Sloan-Kettering Cancer Center; IMRT, intensity-modulated RT.
A significantly longer interval to recurrence was observed for the HIOC group (median, 27.1 months) relative to the comparison group (median, 12.8 months; $P=.0084$; Figure $1, A)$. Among the subgroup of patients who received induction or adjuvant chemotherapy but no radiotherapy, the HIOC group experienced a significantly longer interval to recurrence than did the comparison group (26.3 vs 10 months; $P=.013$; Figure $1, B$ ). However, no significant differences were seen in the interval to recurrence between the HIOC and comparison groups for patients who received radiotherapy in addition to chemotherapy $(P=.14$; Figure $1, C)$. Similarly, the HIOC patients experienced a significantly longer time to recurrence than did the comparison group among the subgroup of patients with pathologic N1 or N2 disease (23.5 vs 11.1 months; $P=.0012$; Figure $1, D)$. The interval to recurrence for patients with pathologic N0 disease was similar for the HIOC and comparison groups $(P=.27$; Figure $1, E)$.

The patients in the HIOC group also had a longer overall survival duration than the patients in the comparison group. The median overall survival was 35.3 months for patients who received HIOC versus 22.8 months for patients who did not receive HIOC $(P=.026$; Figure $2, A)$. The subgroup analysis of overall survival revealed a pattern of findings similar to that observed with the interval to recurrence endpoint. A significantly longer survival duration was observed for the HIOC group than for the comparison group among the patients who had received chemotherapy but no radiotherapy. The median overall survival was 51.1 months for patients in the HIOC group and 20.6 months for those in the comparison group in this subset $(P=.0042$; Figure $2, B$ ). The overall survival for patients who had received radiotherapy in addition to chemotherapy was not significantly different between the HIOC and comparison groups (36.4 vs 31.2 months; $P=.15$; Figure 2, $C$ ). Longer overall survival was observed for the HIOC group than for the comparison group among the patients with pathologic $\mathrm{N} 1$ or N2 disease, with a median overall survival of 33.1 versus 17.4 months, respectively $(P=.018$; Figure $2, D)$. Overall survival did not differ between the 2 groups for those with pathologic N0 disease (36.4 vs 40.3 months; $P=.71$; Figure 2, $E$ ).

In the 2 groups, 9 HIOC patients (13\%) and 7 comparison patients $(23 \%)$ had biphasic histologic findings on the final pathologic analysis (Fisher's exact, $P=.24$ ). Among the patients with biphasic MPM, the median interval to recurrence was 29.2 months for the HIOC group and 12.8 months for the comparison group $(P=.051$; Figure $3, A)$. The median overall survival for the biphasic subgroup was 31.3 months for the HIOC patients and 18.9 months for the comparison patients $(P=.086$; Figure $3, B)$.

Based on the differential effects of HIOC observed among patients treated with and without adjuvant therapy in the subgroup analysis, Cox regression analysis was used 


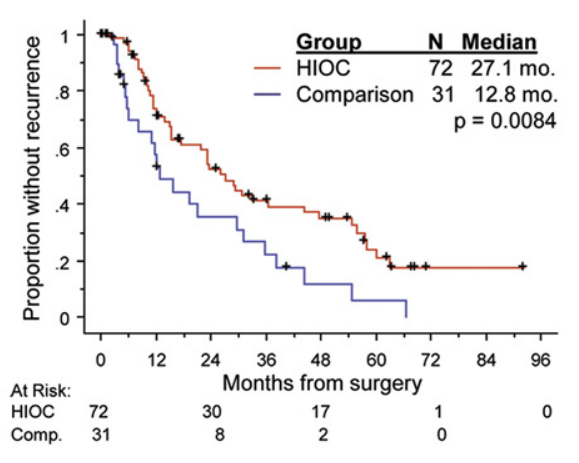

\section{A}
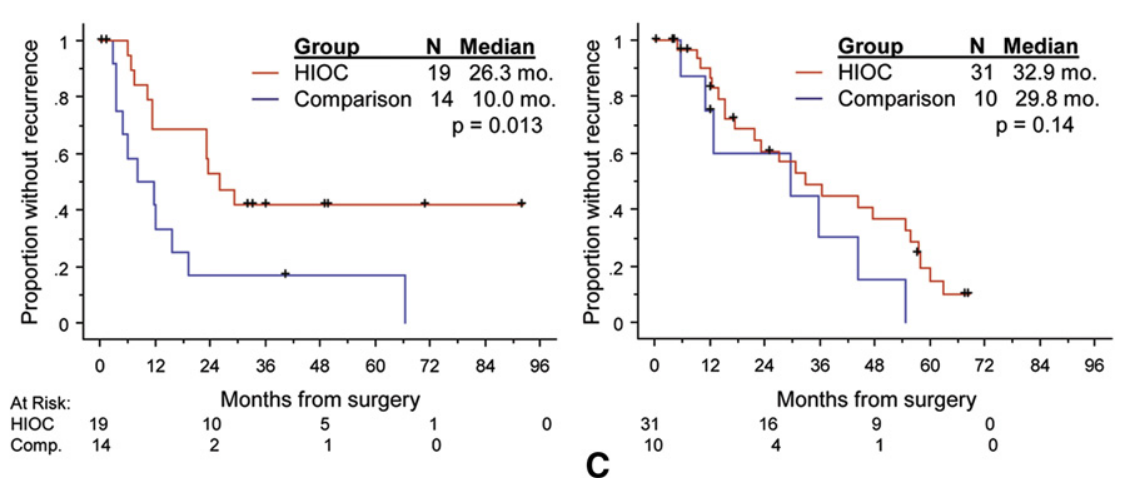

B
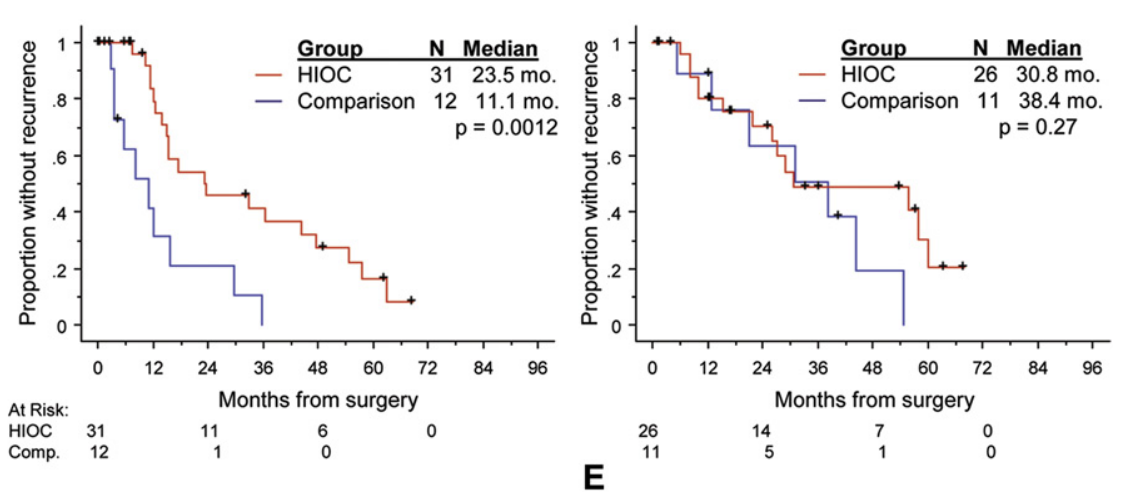

FIGURE 1. Kaplan-Meier survival functions for hyperthermic intraoperative cisplatin chemotherapy (HIOC) and comparison groups depicting interval to recurrence for (A) all patients, (B) patients who received adjuvant or neoadjuvant chemotherapy but no adjuvant radiotherapy, (C) patients who received adjuvant or neoadjuvant chemotherapy and adjuvant radiotherapy, (D) patients with N1 or N2 lymph node metastasis demonstrated on final pathologic examination, and (E) patients with N0 status determined by final pathologic examination.

to directly estimate the effect on overall survival associated with each of the possible combinations of HIOC and adjuvant therapy, with the patients who did not receive either therapy as the reference group. To control for the independent effects of previous therapy and established prognostic factors, the regression model also included neoadjuvant chemotherapy history, histologic findings, and lymph node status. The multivariate analysis (Table 3 ) revealed that a hazard ratio of 0.28 was associated with HIOC followed by adjuvant radiotherapy or chemotherapy, suggesting that the risk of death is reduced by more than $70 \%$ compared with macroscopic complete resection alone. Smaller survival benefits were observed for patients treated with either HIOC or adjuvant therapy but not both; the corresponding hazard ratios of 0.64 and 0.48 suggested an approximately similar benefit for both therapeutic strategies.

\section{DISCUSSION}

With appropriate systemic protection, cisplatin HIOC after surgical macroscopic complete resection is welltolerated by patients and does not increase the hospital length of stay nor interfere with subsequent administration of adjuvant chemotherapy and/or radiotherapy. In this cohort of patients with MPM and preoperative indicators of low-risk disease (epithelial histologic findings on biopsy, 

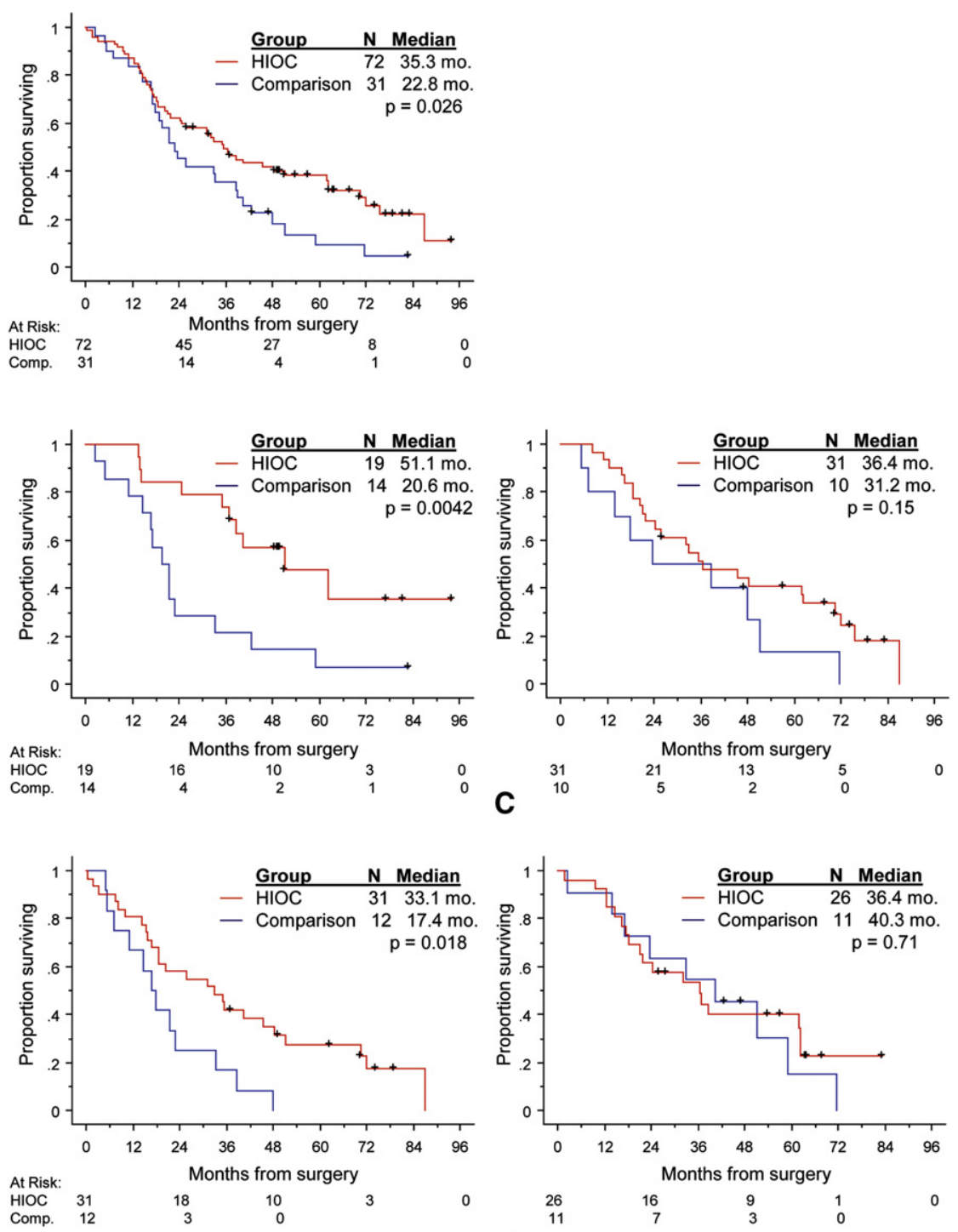

FIGURE 2. Kaplan-Meier survival functions for hyperthermic intraoperative cisplatin chemotherapy (HIOC) and comparison groups depicting overall survival for (A) all patients, (B) patients who received adjuvant or neoadjuvant chemotherapy but no adjuvant radiotherapy, (C) patients who received adjuvant or neoadjuvant chemotherapy and adjuvant radiotherapy, (D) patients with N1 or N2 lymph node metastasis demonstrated on final pathologic examination, and (E) patients with N0 status determined by final pathologic examination.

tumor volume $\leq 500 \mathrm{~cm}^{3}$, and either female gender or male gender with a hemoglobin level within normal limits), treatment with HIOC was associated with an extended interval to recurrence and overall survival.

It is well known that preoperative pleural biopsy has low accuracy for detecting the histologic subtype of MPM. Even when multiple biopsy cores are taken to sample different anatomic areas, the presence of biphasic MPM will sometimes not be detected. ${ }^{15,16}$ Accordingly some patients in the present study had biphasic histologic features demonstrated on the final pathologic examination. Because of the known influence of the histologic type on the outcome endpoints, the potential contribution of the histologic type to the observed effects of HIOC was assessed in a subgroup analysis. The interval to recurrence and overall survival in this very small subgroup both displayed trends consistent with the overall cohort results that approached statistical significance, arguing that the histologic type did not contribute to the observed effects of HIOC. Patients whose tumor histologic type was inaccurately classified from the pleural biopsy findings were likely to have had tumors with a relatively minor sarcomatoid component, which can be as low as $10 \%$ within the classification of biphasic MPM. ${ }^{9}$ Such patients appeared to have a prognosis approaching that of patients with tumors classified as epithelial $(<10 \%$ sarcomatoid component) in this low-risk cohort. 

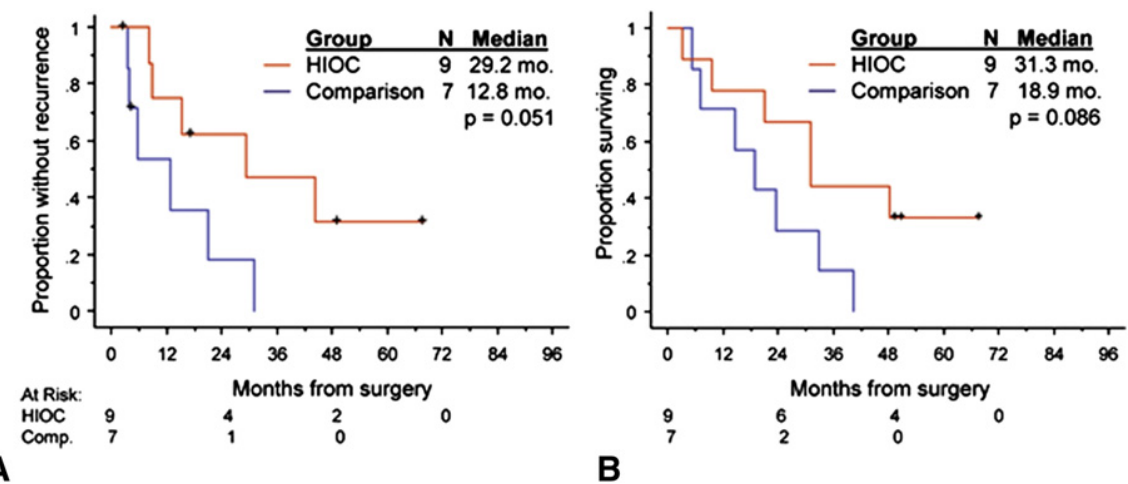

FIGURE 3. Kaplan-Meier survival functions for hyperthermic intraoperative cisplatin chemotherapy (HIOC) and comparison groups depicting (A) interval to recurrence and (B) overall survival for patients found to have biphasic subtype tumors on final pathologic examination.

The comparison group had a greater proportion of patients who received neoadjuvant chemotherapy than did the HIOC group. A number of the patients referred to our program had already been treated with neoadjuvant platinum-based chemotherapy, resulting, in some cases, in deferral of additional intraoperative cisplatin. Because no between-group differences were found in stage distribution or lymph node status, the influence of this imbalance on the outcomes, if any, would be expected to favor the comparison group. No influence of neoadjuvant chemotherapy on overall survival was observed on multivariate analysis (hazard ratio, 1.14).

Even among patients with early MPM who are the most likely to have grossly negative margins at resection, ${ }^{17,18}$ macroscopic complete resection by either extrapleural pneumonectomy or pleurectomy/decortication will, at best, achieve R1 status. This is consistent with the fact that the primary pattern of failure is local within the ipsilateral hemithorax. ${ }^{3}$ Consequently, aggressive systemic and local adjuvant treatment is often delivered to prevent or forestall tumor recurrence. This adjuvant treatment is typically intravenous chemotherapy (after pleurectomy/decortication or extrapleural pneumonectomy) and/or hemithoracic radiotherapy (after extrapleural pneumonectomy only). HIOC represents a method of administering chemotherapy as an additional "local" modality by applying a high concentration of cytotoxic drug to potential microscopic disease at the

TABLE 3. Multivariate analysis estimating effect on overall survival associated with possible combinations of HIOC and adjuvant therapy (patients not receiving either therapy as reference)

\begin{tabular}{lcc}
\hline \multicolumn{1}{c}{ Covariate } & HR & $\mathbf{9 5 \%}$ CI \\
\hline HIOC without adjuvant therapy & 0.64 & $0.25-1.65$ \\
Adjuvant therapy without HIOC & 0.48 & $0.20-1.13$ \\
HIOC with adjuvant therapy & 0.28 & $0.12-0.65$ \\
Neoadjuvant chemotherapy & 1.14 & $0.60-2.17$ \\
Lymph node involvement & 1.69 & $1.02-2.82$ \\
Nonepithelial histologic features & 1.56 & $0.84-2.92$
\end{tabular}

HIOC, Hyperthermic intraoperative cisplatin chemotherapy; $H R$, hazard ratio; $C I$, confidence interval. surface of the retained tissues, including the chest wall, lung (pleurectomy/decortication), and lymph node metastases within the thoracic cavity, with a goal of effectively extending the achieved surgical resection margin by several millimeters.

The beneficial effects of HIOC on the interval to recurrence and overall survival were particularly evident within the subgroup of patients who had not received hemithoracic radiotherapy. By contrast, the incremental benefit of cisplatin HIOC was not significant among patients who underwent extrapleural pneumonectomy with hemithoracic radiotherapy and intravenous chemotherapy, in most cases with a pemetrexed-platinum doublet. Adjuvant radiotherapy might be expected to be more consistently efficacious for local control than HIOC, because it is delivered to the tissues at risk in multiple fractions for an extended period up to a maximal tolerated cumulative dose, instead of a single cisplatin exposure.

However, given the presence of many radiosensitive structures in the hemithorax (ie, heart, esophagus, contralateral lung, spinal cord, small bowel, and liver or stomach, depending on laterality), it is technically very difficult to deliver tumoricidal radiation doses to the entire target of the hemithorax. ${ }^{19}$ Thus, depending on the radiation technique used, significant underdosing of certain areas can result, such as the mediastinum and inferior extent of the costophrenic sulcus. The subgroup of patients with pathologic N1 or N2 lymph node metastases also demonstrated a longer interval to recurrence and overall survival for the HIOC group compared with the comparison group. The latter exhibited a survival duration similar to that previously reported for node-positive patients with epithelial MPM treated with trimodality therapy without HIOC. ${ }^{14}$ By contrast, among patients with lymph node-negative disease, no statistically significant incremental effect of cisplatin HIOC was evident. One could posit that in patients with mediastinal and/or hilar lymph node involvement, radiotherapy might be less effective owing to dose limitations compared with those treated with HIOC, which directly 
treats all structures within the hemithorax at full dose. Accordingly, treatment with HIOC would be associated with incremental local control that will be most apparent for patients with node-positive disease. In support of this interpretation, the multivariate analysis demonstrated synergy between HIOC and adjuvant therapy in reducing the hazard ratio for death.

Thus, the patients who might particularly benefit from HIOC include those with pathologic N1-N2 disease and those who do not receive postoperative radiotherapy. These 2 factors are often not known until after resection. The lymph node status is determined by pathologic review. The decision regarding the delivery of adjuvant radiotherapy depends on many factors. Radiotherapy is often deferred in certain scenarios, such as after pleurectomy, for patients who have experienced postoperative complications or adjuvant chemotherapy-related toxicity, and for those who opt not to receive planned adjuvant radiotherapy. For patients who undergo pleurectomy, full-dose hemithoracic radiotherapy has not been demonstrated to be safe owing to the sensitivity of the retained lung. Among patients who underwent extrapleural pneumonectomy with adjuvant radiotherapy, no significant survival benefit was found for cisplatin HIOC, although the numbers of patients available for subgroup analysis in the present study were too small to draw definitive conclusions.

The decade-long early-phase experience with cisplatin HIOC has enabled us to refine the perioperative management of patients undergoing this treatment. Nephroprotection with fluid administration and the timing of amifostine and sodium thiosulfate has enabled us to minimize the renal toxicity of HIOC. Deep vein thrombosis surveillance has also minimized thromboembolic complications. These adaptations have enabled us to perform cytoreductive surgery with HIOC with morbidity and mortality rates that do not exceed those of cytoreductive surgery alone., ${ }^{4,6}$

The choice of cisplatin for the initial investigation of HIOC was multifactorial. Cisplatin is the most active single agent against mesothelioma, ${ }^{20}$ and it had been successfully administered intrapleurally (although not intraoperatively) by others in previous studies. ${ }^{21,22}$ When administered intravenously, cisplatin has synergistic activity against MPM with doxorubicin, ${ }^{20}$ gemcitabine, ${ }^{23}$ and pemetrexed ${ }^{24}$ and might potentiate other modalities such as radiotherapy and oncolytic viral therapy. ${ }^{25}$ The evidence for efficacy observed in the present study with cisplatin alone encourages additional investigation of this treatment paradigm using combination strategies. Intraoperative lavage provides a basis for locally delivering high-dose combination regimens that might incrementally enhance the efficacy, potentially providing benefit to patients at greater risk and with nonepithelial MPM. A phase I trial at our institution of cisplatingemcitabine HIOC after macroscopic complete resection has recently completed accrual, and assessments of other combination regimens, including cisplatin-pemetrexed, are planned.

The present study was limited by its retrospective nature and small sample size, particularly for the comparison group. The low-risk cohort represented a highly selected group of patients and inherent bias was present in using the group of patients who did not receive HIOC as a comparison group. Moreover, the comparison group included significantly more patients who had received neoadjuvant chemotherapy, limiting the comparability of this cohort to the patients who had received HIOC. The HIOC group included patients treated on and off various protocols during the study period. Patients were able to complete adjuvant therapy with variable consistency, frequently at outside institutions. The inclusion of adjuvant therapy as a covariate in the multivariate analysis was subject to guarantee time bias ${ }^{26}$ which might have resulted in an overestimation of its relative influence on overall survival. These issues introduced some variability and bias into the analysis that could be overcome with a randomized prospective trial. However, a prospective randomized trial is unlikely to be performed. Overall, in evaluating our experience with cisplatin-only HIOC, we have found that our data suggest (but do not prove) that HIOC improves the outcomes of low-risk patients with MPM.

\section{CONCLUSIONS}

Among the low-risk patients, treatment with cisplatin HIOC appears to be particularly beneficial for patients with stage N1-N2 nodal involvement and for those who, for whatever reason, do not undergo adjuvant therapy. Both conditions are difficult to predict preoperatively because of the low sensitivity of the preoperative assessment, imaging, and mediastinoscopy for detecting nodal disease and the inherent difficulties associated with predicting postoperative complications and intolerance to aggressive multimodality therapy. Furthermore, cisplatin HIOC is associated with a favorable toxicity profile. Thus, given the favorable effects on outcomes reported and the minimal incremental morbidity associated with cisplatin HIOC, we recommend incorporation of HIOC into multimodality treatment strategies for patients with low-risk epithelial MPM and without specific contraindications.

The authors sincerely thank Ann S. Adams for expert editorial assistance, and Drs Paul Sugarbaker, John Mannick, Antoon Lerut, Bryan Burt, Brian Goodman, and S. Osman Ali for their critical comments on our report.

\section{References}

1. Sugarbaker DJ. Macroscopic complete resection: the goal of primary surgery in multimodality therapy for pleural mesothelioma. J Thorac Oncol. 2006;1:175-6.

2. Sugarbaker DJ, Flores RM, Jaklitsch MT, Richards WG, Strauss GM, Corson JM, et al. Resection margins, extrapleural nodal status, and cell type determine postoperative long-term survival in trimodality therapy of malignant 
pleural mesothelioma: results in 183 patients. J Thorac Cardiovasc Surg. 1999; 117:54-65.

3. Baldini EH, Recht A, Strauss GM, DeCamp MM Jr, Swanson SJ, Liptay MJ, et al. Patterns of failure after trimodality therapy for malignant pleural mesothelioma. Ann Thorac Surg. 1997;63:334-8.

4. Richards WG, Zellos L, Bueno R, Jaklitsch MT, Janne PA, Chirieac LR, et al. Phase I to II study of pleurectomy/decortication and intraoperative intracavitary hyperthermic cisplatin lavage for mesothelioma. J Clin Oncol. 2006;24:1561-7.

5. Zellos L, Richards WG, Capalbo L, Jaklitsch MT, Chirieac LR, Johnson BE, et al. A phase I study of extrapleural pneumonectomy and intracavitary intraoperative hyperthermic cisplatin with amifostine cytoprotection for malignant pleural mesothelioma. J Thorac Cardiovasc Surg. 2009;137:453-8.

6. Tilleman TR, Richards WG, Zellos L, Johnson BE, Jaklitsch MT, Mueller J, et al. Extrapleural pneumonectomy followed by intracavitary intraoperative hyperthermic cisplatin with pharmacologic cytoprotection for treatment of malignant pleural mesothelioma: a phase II prospective study. J Thorac Cardiovasc Surg. 2009; 138:405-11.

7. Sugarbaker DJ, Wolf AS, Chirieac LR, Godleski JJ, Tilleman TR, Jaklitsch MT, et al. Clinical and pathological features of three-year survivors of malignant pleural mesothelioma following extrapleural pneumonectomy. Eur J Cardiothorac Surg. 2011;40:298-303.

8. Wolf AS, Richards WG, Tilleman TR, Chirieac L, Hurwitz S, Bueno R, et al. Characteristics of malignant pleural mesothelioma in women. Ann Thorac Surg. 2010;90:949-56.

9. Travis WD, Colby TV, Corrin B, Shimosato Y, Brambilla E. World Health Organization international histological classification of tumours: histological typing of lung and pleural tumours. 3rd ed. Berlin: Springer; 1999.

10. Gill RR, Richards WG, Yeap BY, Matsuoka S, Wolf AS, Gerbaudo VH, et al. Epithelial malignant pleural mesothelioma after extrapleural pneumonectomy: stratification of survival with CT-derived tumor volume. AJR Am J Roentgenol. 2012;198:359-63.

11. Gill RR, Yeap BY, Matsuoka S, Wolf AS, Bueno R, Hatabu H, et al. Stage-independent pre-operative prognostic grouping of surgically treated patients with epithelial malignant pleural mesothelioma: 14th World Conference on Lung Cancer, Amsterdam. J Thorac Oncol. 2011;6:S486-7.

12. Rusch VW. A proposed new international TNM staging system for malignant pleural mesothelioma from the International Mesothelioma Interest Group. Chest. 1995;108:1122-8

13. Richards WG. Recent advances in mesothelioma staging. Semin Thorac Cardiovasc Surg. 2009;21:105-10.
14. Sugarbaker DJ, Strauss GM, Lynch TJ, Richards W, Mentzer SJ, Lee TH, et al Node status has prognostic significance in the multimodality therapy of diffuse, malignant mesothelioma. J Clin Oncol. 1993;11:1172-8.

15. Bueno R, Reblando J, Glickman J, Jaklitsch MT, Lukanich JM, Sugarbaker DJ Pleural biopsy: a reliable method for determining the diagnosis but not subtype in mesothelioma. Ann Thorac Surg. 2004;78:1774-6.

16. Kao SC, Yan TD, Lee K, Burn J, Henderson DW, Klebe S, et al. Accuracy of diagnostic biopsy for the histological subtype of malignant pleural mesothelioma. J Thorac Oncol. 2011;6:602-5.

17. Richards WG, Godleski JJ, Yeap BY, Corson JM, Chirieac LR, Zellos L, et al Proposed adjustments to pathologic staging of epithelial malignant pleural mesothelioma based on analysis of 354 cases. Cancer. 2010;116:1510-7.

18. Nakas A, Black E, Entwisle J, Muller S, Waller DA. Surgical assessment of malignant pleural mesothelioma: have we reached a critical stage? Eur J Cardiothorac Surg. 2010;37:1457-63.

19. Baldini EH. Radiation therapy options for malignant pleural mesothelioma Semin Thorac Cardiovasc Surg. 2009;21:159-63.

20. Berghmans T, Paesmans M, Lalami Y, Louviaux I, Luce S, Mascaux C, et al. Activity of chemotherapy and immunotherapy on malignant mesothelioma: a systematic review of the literature with meta-analysis. Lung Cancer. 2002;38: 111-21.

21. Rusch VW, Niedzwiecki D, Tao Y, Menendez-Botet C, Dnistrian A, Kelsen D et al. Intrapleural cisplatin and mitomycin for malignant mesothelioma following pleurectomy: pharmacokinetic studies. J Clin Oncol. 1992;10:1001-6.

22. Lerza R, Esposito M, Vannozzi M, Bottino GB, Bogliolo G, Pannacciulli I. High doses of intrapleural cisplatin in a case of malignant pleural mesothelioma. Cancer. 1994;73:79-84.

23. Byrne MJ, Davidson JA, Musk AW, Dewar J, van Hazel G, Buck M, et al. Cisplatin and gemcitabine treatment for malignant mesothelioma: a phase II study. J Clin Oncol. 1999;17:25-30.

24. Vogelzang NJ, Rusthoven JJ, Symanowski J, Denham C, Kaukel E, Ruffie P, et al Phase III study of pemetrexed in combination with cisplatin versus cisplatin alone in patients with malignant pleural mesothelioma. J Clin Oncol. 2003;21: 2636-44.

25. Adusumilli PS, Chan MK, Chun YS, Hezel M, Chou TC, Rusch VW, et al. Cisplatin-induced GADD34 upregulation potentiates oncolytic viral therapy in the treatment of malignant pleural mesothelioma. Cancer Biol Ther 2006;5:48-53.

26. Anderson JR, Cain KC, Gelber RD. Analysis of survival by tumor response J Clin Oncol. 1983;1:710-9. 